\title{
Von der Diagnose- zur Versorgungskette: Entwicklung der Stufe-3-Leitlinie „Brustkrebs-Früherkennung in Deutschland“
}

\author{
U.-S. Albert, Leitlinienkoordinatorin der S3-Leitlinie „Brustkrebs-Früherkennung“
}

Seit 32 Jahren steht die Brustkrebserkrankung im Zentrum des Tätigkeitsfeldes der Deutschen Gesellschaft für Senologie (DGS). Eine Reihe von Bedarfsanalysen führte 1999 zur Erkenntnis, dass bei steigend hoher Brustkrebsinzidenz die Verfügbarkeit neuer Technologien in Deutschland unzureichend ist, dass strukturelle Defizite mit einer Unausgewogenheit der Gesundheitsversorgung in der Fläche sowie starke Schwankungen in der Versorgungsqualität vorliegen, und dass ein dringender ärztlichmedizinischer Handlungsbedarf besteht, die senologische Gesundheitsversorgung zu verbessern.

Mit Gründung der „Konzertierten Aktion zur Brustkrebs-Früherkennung in Deutschland“ durch Klaus-Dieter Schulz schlossen sich - unter Federführung der DGS - Experten zusammen mit dem Ziel, eine hochqualifizierte Stufe-3-Leitlinie zur Versorgungsverbesserung im deutschen Gesundheitswesen zu erstellen.

Erster Meilenstein war, gemeinsam mit der Arbeitsgemeinschaft Wissenschaftlicher Medizinischer Fachgesellschaften (AWMF), das methodische Vorgehen weiterzuentwickeln. Bis dato waren S3-Leitlinien monodisziplinär und an umschriebene medizinische Probleme eines Sektors ausgerichtet (z.B. die Stufe-3-Leitlinie 1999: „Brennen beim Wasserlassen“ der Deutschen Gesellschaft für Allgemeinmedizin und Familienmedizin). Nunmehr mussten Lösungen für das komplexe Versorgungsproblem „Mammakarzinom“ multidisziplinär und professionell, fach- und sektorübergreifend mit Einbindung der Patientinneninteressen entwickelt werden. Es war ein ungeheueres Anliegen, die Wissenschaft interdisziplinär aus dem Elfenbeinturm herauszuholen, und gebündelte Konzepte zur Verbesserung der Patientinnenversorgung vorzulegen. Es bedeutete, auch in den eigenen Reihen bestehende Barrieren abzubauen. Es galt, eine gemeinsame Arbeitsebene zu finden, die systematisch vorgeht, evidenzbasiert am medizinisch-wissenschaftlich Kenntnisstand ausgerichtet ist, und durch formale Konsensusverfahren die Einbeziehung von Erfahrungswissen ebenso wie Patienteninteressen auf Augenhöhe ermöglicht.

International lagen 1999 insbesondere aus England (SIGN/NICE) Erfahrungen zu komplexen Leitlinien vor, die eine Abschätzung des finanziellen Rahmens eines derartigen Projektes erlaubten, und es war rasch klar, dass nur mit starken Partnern eine Chance auf Realisierung zu erreichen war. Als bis heute stetige Kooperationspartner zur Förderung von S3-Leitlinien bekannten sich die Deutsche Krebshilfe (DKH) und die Deutsche Krebsgesellschaft (DKG). Rückblickend bedurfte es einer Zeitspanne von neun Jahren von der Initiierung der ersten komplexen Stufe-3-Leitlinie durch die DGS, bis zur Etablierung eines finanziell gesicherten "Leitlinienprogramms Onkologie der AWMF, DKG und DKH“ 2008 (www.leitlinienprogramm-onkologie.de).

Die erste strukturierte formale Konsensuskonferenz fand am 24. und 25. Februar 2000 statt. 19 Fachgesellschaften und drei nichtärztliche Organisationen verabschiedeten mit mehr als $90 \%$ Konsens ein 10-Punkte-Programm [1,2]. Der erste Schritt einer evi- denz- und konsensusbasierten Diagnosekette zur BrustkrebsFrüherkennung war gemacht. Die Koordinatoren und 19 Mitglieder der Planungsgruppe führten in 11 Arbeitsgruppen Experten unter Beteiligung von 28 Fachgesellschaften und Berufsverbänden sowie schließlich 4 Patientenorganisationen, die erste komplexe Stufe-3-Leitlinie „Brustkrebs-Früherkennung in Deutschland“ 2003 zur Fertigstellung [3-5]. Das erfolgreiche methodische Konzept wurde für die weitere Versorgungskette Brustkrebs gebündelt. Parallel wurde die Stufe-3-Leitlinie für den Schwerpunkt „Diagnostik, Therapie und Nachsorge des Mammakarzinoms der Frau“ erarbeitet, die 2004 vorlag [6].

Zwei Punkte der Weiterentwicklung der Methodik haben bis heute den Stellenwert von Stufe-3-Leitlinien geprägt:

- Methodische Exaktheit: Recherche, Prüfung und begründete

Bewertung zur Nutzung bereits existierender Leitlinien

- Anwendbarkeit und Überprüfung: Qualitätssicherung und Ableitung von Qualitätsindikatoren

Sie haben Eingang gefunden in das Regelwerk der AWMF und wurden zwischenzeitlich durch die neuen Entwicklungen mit Anforderungen an Aktualisierungen der Leitlinien fortgeschrieben $[7,8]$. Der Lebenszyklus von S3-Leitlinien erfuhr eine Ausgestaltung mit den Elementen Bestandsanalyse (Implementierung, Anwendung und Wirksamkeit mit Erfassung von Qualitätsindikatoren) und Bedarfsanalyse (Priorisierung und Spezifizierung für eine Fortschreibung) zur Erarbeitung der ersten Aktualisierung beider S3-Leitlinien 2008 [9]. Derzeit wird die zweite Aktualisierung durchgeführt. Bis zur Fertigstellung gelten die Stufe-3-Leitlinien von 2008 mit ihren Subdokumenten zur Methodik, den Kurzfassungen und den Frauen- bzw. Patientinneninformationen (www.awmf-leitlinien.de und www.krebshilfe. de) $[10-12]$.

\section{Literatur}

1 Schulz KD. Ärztlich Konzertierte Aktion und 10-Punkte-Programm zur Brustkrebs-Früherkennung in Deutschland. ZaeFQ 2000; 94: 421 - 422

2 Schulz KD, Kreienberg R, Albert US et al. Konzertierte Aktion zur Brustkrebs-Früherkennung in Deutschland. Forum Dt Krebs Ges 2001; 6: $21-25$

3 Albert US, Koller M, Lorenz W et al. Report über die Entwicklung der Leitlinie zur Brustkrebs-Früherkennung in Deutschland: methodisches Vorgehen, Ergebnisse und Implikationen. Gesundh Ökon Qual Manag 2003; 8: 39-51

4 Albert US, Schulz KD, Alt D et al. Eine Leitlinie für Leitlinien: die methodische Entwicklung und Anwendung der Leitlinie Fraueninformation. Zentralbl Gynakol 2003; 125: 484-493 (AWMF Reg. Nr.: 077-02)www. awmf-leitlinien.de

5 Schulz KD, Albert US und die Mitglieder der Arbeitsgruppe „Konzertierte Aktion: Brustkrebs-Früherkennung in Deutschland“. Stufe-3-Leitlinie Brustkrebs-Früherkennung in Deutschland. München: Zuckschwerdt; 2003

6 Kreienberg R, Kopp I, Lorenz W et al. Interdisziplinäre S3-Leitlinie für die Diagnostik und Therapie des Mammakarzinoms der Frau. München: W.Zuckschwerdt; 2004

7 Kopp I, Thole H, Selbmann H et al. Deutsches Instrument zur methodischen Leitlinien-Bewertung (DELBI) Fassung 2005/2006+Domäne 8 (2008). Berlin: AWMF; ÄZQhttp//www.leitlinien.de 2008 
8 Albert US, Kopp I. Was sind eigentlich S1-, S2- und S3-Leitlinien? Gynäkol Prax 2010; 34: 3-9

9 Albert US, Schulz KD, Kopp I. Methodik der S3-Leitlinien-Aktualisierung am Beispiel der Leitlinie „Brustkrebs-Früherkennung in Deutschland“. Prävention und Gesundheitsforschung 2008; 3: 163-172

10 Albert US. Stufe-3-Leitlinie Brustkrebs-Früherkennung in Deutschland 1.Aktualisierung 2008. München: Zuckschwerdt; 2008

11 Kreienberg R, Kopp I, Albert US et al. Interdisziplinäre S3-Leitlinie für die Diagnostik, Therapie und Nachsorge des Mammakarzinoms. München: Zuckschwerdt; 2008: 1

12 Albert US, Schulz KD, Kopp I. Leitlinien-Methodenreport: Aktualisierung der Stufe-3-Leitlinie Brustkrebs-Früherkennung in Deutschland 2007. Marburg:http//www.awmf-leitlinien.de 2007: 1

\section{Bibliografie}

Dol http://dx.doi.org/10.1055/s-0031-1271506

Senologie 2011; 8: 86-87

(c) Georg Thieme Verlag KG Stuttgart · New York · ISSN 1611-6453

\section{Korrespondenzadresse}

\section{Prof. Dr. med. Ute-Susann Albert}

Klinik für Gynäkologie, gynäkologische Endokrinologie und Onkologie Universitätsklinikum Gießen und Marburg GmbH

Standort Marburg Brustzentrum Regio

Baldingerstraße

35043 Marburg

albertu@med.uni-marburg.de 